\title{
Predicting Surgical Resource Consumption and In- hospital Mortality in Resource-scarce Conflict Settings: A Retrospective Database Study
}

Måns Muhrbeck ( $\square$ mans.muhrbeck@liu.se )

Vrinnevi Hospital https://orcid.org/0000-0001-7002-7768

Zaher Osman

International Committee of the Red Cross

Johan Von Schreeb

Karolinska Institute: Karolinska Institutet

Andreas Wladis

Linköping University: Linkopings universitet

Peter Andersson

Linköping University: Linkopings universitet

Research article

Keywords: Armed Conflicts, Health Resources, Penetrating Wounds

Posted Date: January 7th, 2021

DOI: https://doi.org/10.21203/rs.3.rs-140019/v1

License: (c) (1) This work is licensed under a Creative Commons Attribution 4.0 International License.

Read Full License

Version of Record: A version of this preprint was published at BMC Emergency Medicine on August 11th, 2021. See the published version at https://doi.org/10.1186/s12873-021-00488-2. 


\section{Predicting surgical resource consumption and in-hospital mortality in resource-scarce conflict settings: a retrospective database study}

\section{Authors}

Måns Muhrbeck ${ }^{1,2}$, Zaher Osman ${ }^{3}$, Johan von Schreeb ${ }^{4}$, Andreas Wladis ${ }^{2,5}$ and Peter Andersson ${ }^{5,6}$

\section{Affiliations}

${ }^{1}$ Division of Surgery, Department of Biomedical and Clinical Sciences, Linköping University, Norrköping, Sweden

${ }^{2}$ Center for Teaching and Research in Disaster Medicine and Traumatology, Linköping University, Linköping, Sweden

${ }^{3}$ International Committee of the Red Cross, Geneva, Switzerland

${ }^{4}$ Department of Global Public Health, Karolinska Institutet, Stockholm, Sweden

${ }^{5}$ Department of Biomedical and Clinical Science, Linköping University, Linköping, Sweden

${ }^{6}$ International Medical Programme, Center for Teaching and Research in Disaster Medicine and Traumatology, University Hospital, Linköping, Sweden

\section{Corresponding author}

Måns Muhrbeck, MD, mans.muhrbeck@liu.se

Department of Surgery, Vrinnevi Hospital

S-603 79 Norrköping, Sweden,

$\mathrm{T}:(+4610) 1040000$

F: (+46 10) 1043188

\section{Manuscript category}

Observational studies 


\section{Based on previous communication to a society or meeting}

Preliminary results were presented orally at the Swedish Surgical Society annual meeting, August 22-26, 2016, Malmö, Sweden, and at the $6^{\text {th }}$ World Society of Emergency Surgery annual meeting, June 26-28 June, 2019, Nijmegen, the Netherlands, and an abstract was orally presented at the $20^{\text {th }}$ WADEM Congress on Disaster and Emergency Medicine, April 2528, 2017, Toronto, Canada. 


\section{Abstract}

Background: In armed conflicts, civilian health care struggles to cope. Being able to predict what resources are needed is therefore vital. The International Committee of the Red Cross (ICRC) implemented in the 1990s the Red Cross Wound Score (RCWS) for assessment of penetrating injuries. It is unknown to what extent RCWS or the established trauma scores Kampala trauma Score (KTS) and revised trauma score (RTS) can be used to predict surgical resource consumption and in-hospital mortality.

Methods: A retrospective study of routinely collected data on weapon-injured adults admitted to ICRC's hospitals in Peshawar, 2009-2012 and Goma, 2012-2014. High resource consumption was defined as $\geq 3$ surgical procedures, $\geq 3$ blood-transfusions and/or amputation. The relationship between RCWS, KTS, RTS and resource consumption, in-hospital mortality was evaluated with logistic regression and adjusted receiver operating characteristic curves (AUC). The impact of missing data was assessed with imputation. Model fit was compared with Akaike Information Criterion (AIC).

Results: A total of 1564 patients were included, of these 834 patients had complete data. For high resource consumption AUC was significantly higher for RCWS (0.76) than for KTS (0.53) and RTS (0.51). Additionally, RCWS had lower AIC, indicating a better model fit. For in-hospital mortality AUC was significantly higher for RCWS (0.79) than for KTS (0.71) and RTS (0.70) for all patients, but not for patients with complete data.

Conclusion: RCWS appears to predict surgical resource consumption better than KTS and RTS. RCWS may be a promising tool for planning and monitoring surgical care in resource-scarce conflict settings.

Keywords: Armed Conflicts; Health Resources; Penetrating Wounds 


\section{Manuscript}

\section{Introduction}

In armed conflicts, civilian causalities often must rely on frail and fragmented health care systems that can only offer limited or no surgical resources to treat injured with potentially life-threatening injuries[1]. For healthcare providers delivering surgical care in conflicts it is therefore imperative to know how to use available surgical resources as efficiently as possible. Surgical resource consumption or workload in conflicts has in previous studies been discussed in terms of the number of surgical procedures, amputations, use of blood products, and mortality rate[2-7]. However, to our knowledge no consistent method to assess surgical resource consumption exists. Trauma scores used in non-conflict settings could potentially be used for this purpose. The commonly registered vital sign-based Revised Trauma Score (RTS) has been demonstrated to correlate with the need for surgery, haemorrhage, and mortality[811]. In resource-scarce settings the Kampala Score (KTS), a simplified combination of age, RTS and the anatomical-based Injury Severity Score (ISS), developed by Kobusingye et al has been validated as being able to predict the need for admission and mortality[12]. However, the predictive abilities for these scores could be diminished in conflicts due to disintegrated infrastructure, lack of transportation means, non-existing prehospital care and long distance to hospital that cause a survival bias where injured with life-threatening, but treatable, injuries die before reaching the hospital.

The International Committee of the Red Cross (ICRC) is a humanitarian organization providing medical assistance to victims of war and other situations of violence, including independently running or supporting hospitals. In the early 1990s Mr Robin Coupland, at the time Chief surgeon for ICRC, developed a system for wound classification (Red Cross Wound Score, RCWS) in order to help surgeons systematically classify and triage penetrating wounds[13]. To 
classify a wound according to RCWS, the size of the wound (RCWS grade) and type of tissue involved (RCWS type) must be assessed (Table 1). The grade directly translates into the degree of tissue damage at injury[4, 13, 14].

The aim of the present study was to determine if RCWS, KTS and RTS have predictive ability to assess surgical resource consumption and in-hospital mortality in resource-scarce conflict settings. Surgical resource consumption was characterized by the number of surgical procedures, blood transfusions and limb amputation. A secondary aim was to assess if the individual components of RCWS are independent predictors of surgical resource consumption and in-hospital mortality. If either of these scores could be found to be reliable they would provide an urgently needed instrument for planning and monitoring surgical interventions in resource-scarce conflict settings.

\section{Patients and Settings}

Routinely collected patient data from ICRC's hospitals in Peshawar, Pakistan, and Goma, Democratic Republic of the Congo (DRC), was retrospectively analysed for this study. Patients treated at ICRC's hospital in Peshawar were predominantly injured in the conflict on both sides of the Pakistani-Afghanistan border. The hospital was closed in 2014 and had 116 beds, a critical care unit, three operating theatres, $\mathrm{X}$-ray services and a laboratory[15]. Patients treated at the hospital in Goma were mainly injured in the ongoing conflict between several rivalling factions in the Kivu province in the DRC. Compared to the conflict along the Afghanistan-Pakistani border, fewer injures from indiscriminate weapons such as grenades, bombs and anti-personnel mines have been reported[2, 15, 16]. The hospital is still operational and has 65 beds, one operating theatre, X-ray services and a laboratory. Both hospitals had access to physiotherapy for post-operative mobilization and rehabilitation with prosthesis fitting[15]. 
At both hospitals combatants as well as local citizens with weapon-related injuries were treated. Those seeking care were not asked whether they were civilians or combatants. Care at both hospitals was provided free of charge during the study period.

\section{Method}

The material consists of two matching databases of anonymized patient data from the ICRC's hospitals in Peshawar and Goma. All patients treated between 18 February 2009-9 May 2012 (Peshawar) and 17 November 2012-17 September 2014 (Goma) were continuously included in the two databases (Excel ${ }^{\circledR}$ spreadsheet) at the time of patient discharge. Entries in the Peshawar database were done by two physicians whose primary job was to maintain the database. Entries in the Goma database were done by health care staff after receiving basic training. In total 3028 patients were recorded in the Peshawar database and 689 in Goma database. The two databases were merged and transferred to SPSS Statistical software version 25 (IBM Corporation, Armonk, NY, USA) for this study. The final database was validated with respect to the original databases by comparing data from 50 patients from each database without any differences identified. Patients that fulfilled the following inclusion criteria were extracted from the merged database: adult patients with weapon-related penetrating injuries, assessed RCWS and known discharge status. Adults were defined as age 15 or older in accordance with previous studies from similar settings[15, 17]. RCWS was determined by the surgeon at the time of the first surgical procedure according to ICRC guidelines[4]. Surgeons had received training in the use of RCWS prior to deployment. The wound with the highest RCWS grade and type was recorded as the first injury. To categorize a penetrating injury according to RCWS, the wound's grade 1-3 (wound's entry- and exitdiameter and if a two-fingers- wide cavity exists) and type (soft tissue, bones, threatening life or threatening life or limb) needs to be determined (Table 1). Depending on the grade and type, a wound is assigned to one of twelve different categories (Supplement Table 1)[4, 13, 
18]. The presence of a second severe injury was dichotomously registered as existing or not. Severe injury was defined as a cavitating soft tissue loss of $10 \mathrm{~cm}$ or more and/or open fracture and/or injury to vital structure. This definition excludes RCWS soft tissue injury grade 1 and 2 and translates to an Abbreviated Injury Score of 2 or above for any given anatomical site, corresponding to the definition used in KTS[19]. In the case of multiple admissions only, the first one was included. Patients with unspecified gender, time of injury and vital signs were considered to be incomplete cases and excluded in the primary analysis (Figure 1).

After discussion with senior ICRC-surgeons and analysis of previously published studies, we concluded that the number of surgical procedures under anaesthesia, blood transfusions and limb amputation would best reflect surgical resource consumption in the resource-scarce conflict settings where the ICRC works[2-5, 7, 20]. High surgical resource consumption was dichotomously defined as: three or more surgical procedures under anaesthesia, three or more blood transfusions or in-hospital amputation proximal to the metacarpophalangeal joints of the hand or the midtarsal joints of the foot. Three or more surgical procedures were chosen because a study with 16172 patients treated at ICRC's hospitals demonstrated that $66 \%$ of the patients were treated with two surgical procedures or fewer[4]. Three or more blood transfusions was chosen as a measure because a previous ICRC-study with 4470 weapon-injured patients demonstrated that $15.5 \%$ were transfused and an average of 2.9 units was given[20]. Furthermore, the ICRC recommends an availability of 100 units/100 patients if anti-personnel mines are used in the conflict[4]. In-hospital limb amputation was chosen because these patients require extensive resources postoperatively in terms of physiotherapy and prosthesis fitting. In-hospital mortality was defined as deceased during hospital stay. 


\section{Statistical methods}

We estimated that 592 patients would be needed to detect a difference of 0.10 in the trauma scores' ability to detect high surgical resource consumption. A reference AUC of $0.75, \mathrm{Cl} 95 \%$ and power $90 \%$ was used for this sample size calculation. Nonetheless, all eligible patients were included in the analysis.

Descriptive comparisons between Peshawar and Goma were analysed using Fisher's exact test for categorical variables and Mann-Whitney $U$-test for continuous variables. Values are given as mean with standard deviation (SD) where appropriate. To compare the predictive effect of the individual components of RCWS, KTS and RTS on surgical resource consumption and inhospital mortality, binary logistic regression was performed. Gender, age (15-49 and >49 yrs.), time since injury $(0-6,7-24$ and $>24 \mathrm{~h})$ systolic blood pressure $(0-75,76-89$, and $>89 \mathrm{~mm} \mathrm{Hg})$, respiratory rate $(\leq 9,10-29, \geq 30 / \mathrm{min})$, Glasgow Coma Scale $(\leq 5,6-8,9-12$ and $13-15)$, RCWS wound grade, RCWS wound type and the existence of more than one severe injury were predictors included in the regression analysis as these are the components of RCWS, KTS and RTS or have in previous studies been identified as influencing the need for surgical intervention, blood transfusion or amputation [2, 21-24]. Vital signs were taken upon admission, and the intervals used match the intervals used in RTS[8]. The procedure to calculate RTS and KTS has been described elsewhere[8, 12]. Forward Wald was used to calculate a final regression model. When comparing differences in resource consumption for the Peshawar and Goma hospital, hospital site was added to the final regression model. The impact of predictors is presented as an odds ratios (OR) with $95 \%$ confidence intervals. Pvalues (two-tailed) less than 0.05 were considered significant for both the univariate and logistic regression analysis.

The sensitivity and specificity associated with the ability of RCWS, KTS and RTS to predict high surgical resource consumption and in-hospital mortality was assessed by analysing the areas under the receiver operating characteristic curve (AUC). To enable analysis of patients with 
more than one severe injury, RCWS was adjusted for the existence of several severe injuries. In making comparisons of the prognostic abilities of RCWS, KTS and RTS regarding high surgical resource consumption and in-hospital mortality the AUCs along with $95 \%$ confidence intervals (Cl) was used. Overlapping confidence intervals were interpreted as indicating non-significant differences in the prognostic abilities of RCWS, KTS and RTS.

Comparison of goodness of model fit for RCWS, KTS and RTS in predicting high surgical resource consumption and in-hospital mortality was analysed by Akaike Information Criterion (AIC). A lower AIC score for a trauma score indicates a better fitting model in comparison with the other evaluated trauma scores. Little's MCAR test and imputation with fully conditional specification was done to enable the analysis of incomplete cases and thereby assessing the impact of exclusion of patients with unknown gender, time of injury and vital signs. MannWhitney U-test with Fisher's exact test was used to evaluate why a patient ended up in the high resource group. 


\section{Results}

\section{Descriptive}

A total of 657 of 1324 patients treated in Peshawar and 177 of 240 patients treated in Goma had complete data (Complete cases, Figure 1). In Peshawar a larger proportion were men (Peshawar: 589 patients, 90\%, and Goma: 149 patients, 84\%, $\mathrm{p}=0.047$ ) and more patients were older than 49 years compared to Goma (Peshawar: 67 patients, 10\% and Goma: 5 patients, 3\%, $p<0.001$ ). There was no difference in mean age (Peshawar: 29.1 years, SD 12.0, and Goma: 29.6 years, SD 9.8, $\mathrm{p}=0.617$ ). More patients were treated for injuries related to indiscriminate weapons such as bombs, shells and fragments or anti-personnel mines and fewer related to gunshots in Peshawar than in Goma ( $p<0.01$ for all) (Table 2$)$. Fewer patients were admitted within 6 hours of the injury in Peshawar compared to Goma (Peshawar: 1 patient, $0.2 \%$ and Goma: 35 patients, 20\%, $\mathrm{p}<0.00001$ ). Comparisons of injury patterns between the hospitals are shown in Table 3. There was no difference in the RCWS wound type for first injury recorded between the hospitals. Patients with injuries to the head, face or having more than one severe injury were more frequent in Peshawar, while injuries to the upper limb were less frequent compared to Goma ( $p<0.05$ for all). Surgical care received and in-hospital mortality are presented in Table 4. Chest-tube insertion and fracture surgery were more frequent in Peshawar than in Goma ( $p=0.039$ and $p<0.0001$, respectively). There was no difference in frequencies of amputations, blood transfusions and in-hospital mortality between the hospitals.

\section{Surgical resource consumption}

The relationship between surgical resource consumption, vital signs and wound characteristics are shown in Table 5. A larger portion of the patients with high surgical resource consumption had lower systolic blood pressure, higher RCWS wound grade and type and more frequently had several severe injuries compared to patients with low surgical 
resource consumption ( $p<0.01$ for all). When adjusting for all predictors in the final logistic regression model RCWS wound grade, RCWS wound type and existence of several severe injuries remained significant. Gender, age, time since injury, systolic blood pressure, respiratory rate and Glasgow Coma Scale did not affect a patient's surgical resource consumption.

Analysis of the area under receiver operating characteristic curve (AUC) and Akaike Information Criterion (AIC) for respective trauma score for high surgical resource consumption are shown in Table 6 and Figure 2. For complete cases RCWS achieved significantly higher AUC and lower AIC, i.e. better predictive ability and model fit, than KTS and RTS for surgical resource consumption.

In the logistic regression model for all cases, using imputed data for incomplete cases, the same predictors and also age more than 49 years ( $\mathrm{OR} \mathrm{1.84,95 \%} \mathrm{Cl} 1.26-2.70, \mathrm{p}<0.001$ ) were found to be significant (Supplement Table 2). The analysis of AUC for all cases was basically unchanged when compared to the analysis of complete cases (Table 6 and Figure 2).

\section{Subgroup analysis of high resource consumption and hospital site}

When hospital site was added to the final logistic regression model of complete cases, patients treated in Goma were more likely to have a high surgical resource consumption than patients treated in Peshawar (OR 2.01, 95\% Cl 1.36-2.98, $\mathrm{p}<0.001$ ). After adjusting surgical procedures shown in table 3 for the total number of injured sites, patients in Goma were more frequently exposed to soft tissue surgery (Goma: mean 3.58 times/patient, SD 3.39, and Peshawar: 2.18, SD 1.75, $\mathrm{p}<0.00001$ ) and also to surgical procedures under anaesthesia than in Peshawar (Goma: mean 3.71 times/patient, SD 3.71, and Peshawar: 1.96, SD 1.55, $p<0.00001$ ). Patients in Goma were subjected to fracture surgery to a lesser extent than in Peshawar (Goma: mean 0.13 times/patient, SD 0.44, and Peshawar: 0.21, SD 0.46, $p=0.0002$ ). There was no difference in amputation rates (Goma: mean 0.03 amputations/patient, SD 0.16, and Peshawar: 0.03, SD 
0.15, $p=0.540$ ) and blood transfusions (Goma: mean 0.34 units/patient, SD 1.19, and Peshawar: 0.19, SD 0.56, $\mathrm{p}=0.521)$. When comparing Goma and Peshawar regarding patients with high surgical resource consumption the distribution between hospitals was similar with regard to the number of surgical procedures, blood transfusions and amputation rates. Three or more surgical procedures most strongly explained why most patients ended up in the high resource consumption group for both hospital sites (Goma: 86 of 102 patients, $84.3 \%$, and Peshawar: 197 of 260 patients, $75.8 \%, p=0.090)$.

\section{In-hospital mortality}

There was no difference in in-hospital mortality between the hospital sites (Peshawar: 18 patients, 2.7\% and Goma: 3 patients, 1.7\%, $p=0.781$ ). A larger portion of the patients that died during hospital stay arrived sooner after injury, had lower systolic blood pressure, lower GCS and higher RCWS type compared to patients that were discharged alive ( $p<0.05$ for all) (Supplement Table 3). In the logistical regression model for complete cases no predictors were significant for in-hospital mortality. Analysis of the area under receiver operating characteristic curve (AUC) and Akaike Information Criterion (AIC) for individual patient's trauma score for in-hospital mortality are shown in Table 7 and Fig 3. RCWS predictive ability for in-hospital mortality was not significantly better than KTS and RTS.

In the logistic regression model for all cases, using imputed data for incomplete cases, systolic blood pressure 76-89 mmHg and RCWS wound types threatening life and threatening life or limb were found to be significant predictors for in-hospital mortality (systolic blood pressure 76-89 mmHg: OR 4.63, 95\% Cl 1.59-13.44, threatening life: OR 10.72, 95\% 4.42-26.01 and threatening life or limb: $8.43,95 \% \mathrm{Cl} 3.36-21.18, \mathrm{p}<0.01$ for all) (Supplement Table 3). In the analysis of AUC for all cases RCWS demonstrated a significantly higher AUC and lower AIC, i.e. better predictive ability and model fit, than KTS and RTS for in-hospital mortality, (Table 7 and Fig 3). 


\section{Discussion}

This is to our knowledge the first study to demonstrate that RCWS could have a predictive ability for surgical resource consumption in adult patients with weapon-related injuries. The findings are consistent with previous studies that have demonstrated an association between RCWS and the need for surgery, in-hospital amputation and blood transfusions[25-27]. Furthermore, in a previous study from our group findings suggest that RCWS correlates with number of surgeries, blood transfusions and hospital stay in paediatric patients with weaponrelated extremity wounds[28]. When comparing RCWS with KTS and RTS, commonly used trauma scores in non-conflict settings, RCWS had a better ability to predict surgical resource consumption. This might not be surprising since both the former scores were designed to predict mortality and not surgical resource consumption[8, 12]. However, to our knowledge, no instrument or trauma score has yet been validated for measurement of surgical resource use in conflict settings. KTS and RTS were chosen as they are commonly used trauma scores and have been shown to correlate with resource-related outcomes in trauma settings [10-12, $29,30]$. Furthermore, RCWS, KTS and RTS are similar in the aspect that they can be assessed without the need of advanced diagnostic equipment or calculations. A source of error is that we had a large number of missing vital signs in our dataset potentially affecting the predictive abilities for KTS and RTS. To address this issue, we performed the logistic regression model with imputed data for missing vital signs without any substantial changes in vital signs impact of surgical resource consumption.

Patients treated in Peshawar were more frequently injured by indiscriminate weapons, arrived later to the hospital, more frequently had injuries to the head or face and more often had more than one severe injury than in Goma. Therefore, it was unexpected to find that the patients in Goma were more frequently exposed to soft tissue surgery and surgery under anaesthesia than in Peshawar. The reason for the difference in surgical resource consumption 
is unclear as both hospitals followed the same treatment protocol and were similarly equipped. A plausible explanation could be that patients treated in Goma were in a poorer general condition with impaired healing ability due to being more frequently affected by malnutrition or endemic diseases such as tuberculosis and malaria compared to Peshawar[31]. This difference in consumption of surgical resources between Goma and Peshawar illustrates how RCWS can be used to systematically asses treatment facilities use of surgical resources in relation injuries treated.

Consistent with other studies from armed conflicts, a low in-hospital mortality was observed at both hospitals[15, 32]. In the logistic regression analysis of complete cases none of the components of RCWS, KTS and RTS were found to be significant predictors for in-hospital mortality resulting in AUC values with large and overlapping confidence interval. In the analysis of all cases systolic blood pressure 76-89, RCWS wound types threatening life and threatening life or limb were found to be significant predictors for in-hospital mortality rendering significantly better predictive ability for RCWS than KTS and RTS. The grade component of RCWS has previously been found to correlate with mortality in patients with conflict-related abdominal wounds with penetration of the peritoneum[33]. Otherwise little has previously been known regarding RCWS predictive ability for mortality. In non-conflict settings better predictive abilities regarding weapon-related injuries have been reported for KTS and RTS[34, 35]. The worse predictive ability observed in our study could be explained by the survival bias where patients with the most altered vital signs die before reaching the hospital. The low in-hospital mortality and that $93.3 \%$ of the patients in our material arrived more than six hours after the injury occurred supports this assumption. Consequently, the early, but potentially preventable, deaths in the trimodal distribution of trauma death proposed by Trunkey is not seen in our material[4, 36]. The conclusion that RCWS has better 
predictive ability for in-hospital mortality than KTS and RTS in conflicts must therefore be made with caution.

The difficulty to foresee what resources will be needed in conflicts coupled with fragile and unreliable supply chains can potentially lead to disastrous effects in terms of morbidity and mortality $[4,37]$. Our findings support the use of RCWS as an instrument for evaluation of surgical resource needs and in-hospital mortality in relation to injuries treated. RCWS could consequently be used to facilitate that the surgical resources supplied meet the actual treatment needs. The systematic use of RCWS could also enable quality audits and research in conflict settings. The inclusion of RCWS in the minimum data set recommended by the Consensus Framework for the Humanitarian Surgical Response to $21^{\text {st }}$ Century Warfare should therefore be considered[38]. However, prospective studies from different armed conflicts are needed to assess the external validity of our findings and determine the predictive ability for each RCWS category. Furthermore, the interobserver reliability for RCWS needs to be evaluated.

\section{Limitations}

The use of routinely collected patient data limits the possibility of controlling for potentially confounding factors, such as variations in workload, adherence to treatment protocols, access to drugs, blood products, disposable material and missing data. To mitigate this issue, we also analysed the predictive abilities for RCWS, KTS and RTS with all patients treated for weaponrelated injuries during the given time periods at both hospital sites using an imputed dataset for missing variables with only minor alterations in the results. Nevertheless, alternative explanations for our findings could exist. A prospective cohort study design would have allowed for better control of confounding factors. However, prospective data collection in conflict settings is often difficult due to restraints in infrastructure and frequent rotation of health care staff. Additionally, participation in a study can have negative implications for 
participating health care staff and patients. In this study, we had good control of how the data was collected.

\section{Conclusion}

Our findings indicate that RCWS was able to predict high surgical resource consumption better than and in-hospital mortality at least equal to KTS and RTS. In addition to facilitating systematic evaluation of penetrating injuries RCWS could consequently be a useful instrument in planning and monitoring of surgical care facilities in resource-scarce conflict settings. 


\section{List of Abbreviations}

AIC: Akaike Information Criterion, AUC: Area under the receiver operating characteristic curve, $\mathrm{Cl}$ : Confidence interval, ICRC: International Committee of the Red Cross, ISS: Injury Severity Score, KTS: Kampala Trauma Score, OR: Odds ratio, RCWS: Red Cross Wound Score, RTS: Revised Trauma Score and SD: Standard deviation.

\section{Acknowledgements}

Open access funding provided by Linköping University. We thank former ICRC chief surgeons Robin M. Coupland and Harald Veen for providing indispensable input on how to define surgical resource consumption and Lars Valter, Forum Östergötland, Linköping University, Sweden, for statistical advice. Funding was provided by RALF and ALF grants, Region Östergötland, Sweden.

\section{Author contribution}

This study was conceived and designed by MM, JvS and PA. Data were collected by ZO in Peshawar and ICRC-staff in Goma and analysed by MM and PA. Interpretation of the data was done by MM, ZO, JvS, AW and PA. The first draft of the manuscript was made by MM and was critically revised by all authors.

\section{Funding}

RALF and ALF grants (grant numbers 06000908 and LIO-79962), Region Östergötland, Sweden.

\section{Availability of data and materials}

The datasets used and/or analysed during the current study are available from the corresponding author on reasonable request. 


\section{Ethics approval and consent to participate}

The study was approved by the ICRC. Exploration of data was approved by the Regional Ethical Review Board in Linköping, Sweden (Dnr. 2014/417-31, 2015/66-33, 2016/298-32).

\section{Consent for publication}

Publication of the study was approved by the ICRC.

\section{Competing interests}

The authors have no conflicts of interest to declare. ZO has a long-term contract at the ICRC, AW was Chief Surgeon at the ICRC 2017-2019 and PA has had several intermittent contracts with the ICRC.

\section{Author details}

${ }^{1}$ Division of Surgery, Department of Biomedical and Clinical Sciences, Linköping University, Norrköping, Sweden. ${ }^{2}$ Center for Teaching and Research in Disaster Medicine and Traumatology, Linköping University, Linköping, Sweden. ${ }^{3}$ International Committee of the Red Cross, Geneva, Switzerland. ${ }^{4}$ Department of Global Public Health, Karolinska Institutet, Stockholm, Sweden. ${ }^{5}$ Department of Biomedical and Clinical Sciences, Linköping University, Linköping, Sweden. ${ }^{6}$ International Medical Programme, Center for Teaching and Research in Disaster Medicine and Traumatology, University Hospital, Linköping, Sweden. 


\section{References}

1. Leaning J, Guha-Sapir D: Natural disasters, armed conflict, and public health. N Eng/ J Med 2013, 369(19):1836-1842.

2. Andersson P, Muhrbeck M, Veen H, Osman Z, von Schreeb J: Hospital Workload for Weapon-Wounded Females Treated by the International Committee of the Red Cross: More Work Needed than for Males. World J Surg 2018, 42(1):93-98.

3. Gertsch P: Assessment of hospital workload in war surgery. Br J Surg 1987, 74(9):831-833.

4. Giannou C, Baldan M, Comité international de la Croix-Rouge: War surgery : working with limited resources in armed conflict and other situations of violence. Geneva: ICRC; 2019.

5. Coupland RM, Korver A: Injuries from antipersonnel mines: the experience of the International Committee of the Red Cross. BMJ 1991, 303(6816):1509-1512.

6. Ramalingam T: Extremity injuries remain a high surgical workload in a conflict zone: experiences of a British Field Hospital in Iraq, 2003. JRAMC 2004, 150(3):187-190.

7. Korver AJ: Injuries of the lower limbs caused by antipersonnel mines: the experience of the International Committee of the Red Cross. Injury 1996, 27(7):477-479.

8. Champion HR, Sacco WJ, Copes WS, Gann DS, Gennarelli TA, Flanagan ME: A revision of the Trauma Score. J Trauma 1989, 29(5):623-629.

9. Roy N, Gerdin M, Schneider E, Kizhakke Veetil DK, Khajanchi M, Kumar V, Saha ML, Dharap S, Gupta A, Tomson G et al: Validation of international trauma scoring systems in urban trauma centres in India. Injury 2016, 47(11):2459-2464.

10. Emerman CL, Shade B, Kubincanek J: A comparison of EMT judgment and prehospital trauma triage instruments. J Trauma 1991, 31(10):1369-1375.

11. Raux M, Sartorius D, Le Manach Y, David JS, Riou B, Vivien B: What do prehospital trauma scores predict besides mortality? J Trauma 2011, 71(3):754-759.

12. Kobusingye OC, Lett RR: Hospital-based trauma registries in Uganda. J Trauma 2000, 48(3):498-502.

13. Coupland RM: The Red Cross classification of war wounds: the E.X.C.F.V.M. scoring system. World J Surg 1992, 16(5):910-917.

14. Cernak I, Savic J, Zunic G, Pejnovic N, Jovanikic O, Stepic V: Recognizing, scoring, and predicting blast injuries. World J Surg 1999, 23(1):44-53.

15. Haverkamp FJC, van Gennip L, Muhrbeck M, Veen H, Wladis A, Tan E: Global surgery for paediatric casualties in armed conflict. World J Emerg Surg 2019, 14:55.

16. Chu K, Havet P, Ford N, Trelles M: Surgical care for the direct and indirect victims of violence in the eastern Democratic Republic of Congo. Confl Health 2010, 4:6.

17. Wagner Z, Heft-Neal S, Wise PH, Black RE, Burke M, Boerma T, Bhutta ZA, Bendavid E: Women and children living in areas of armed conflict in Africa: a geospatial analysis of mortality and orphanhood. Lancet Glob Health 2019, 7(12):e1622-e1631.

18. Coupland RM, Comité international de la Croix-Rouge. Division médicale: The Red Cross wound classification. Geneva: International Committee of the Red Cross; 1993.

19. MacLeod JBA, Kobusingye O, Frost C, Lett R, Kirya F, Shulman C: A Comparison of the Kampala Trauma Score (KTS) with the Revised Trauma Score (RTS), Injury Severity Score (ISS) and the TRISS Method in a Ugandan Trauma Registry. Eur J Trauma 2003, 29(6):392-398.

20. Eshaya-Chauvin B, Coupland RM: Transfusion requirements for the management of war injured: the experience of the International Committee of the Red Cross. $\mathrm{Br} J$ Anaesth 1992, 68(2):221-223. 
21. Forrester JD, Forrester JA, Basimouneye JP, Tahir MZ, Trelles M, Kushner AL, Wren SM: Sex disparities among persons receiving operative care during armed conflicts. Surgery 2017, 162(2):366-376.

22. Azad AD, Kong VY, Clarke DL, Laing GL, Bruce JL, Chao TE: Use of vital signs in Predicting surgical intervention in a South African population: A cross-sectional study. Int J Surg 2020.

23. Kotwal RS, Howard JT, Orman JA, Tarpey BW, Bailey JA, Champion HR, Mabry RL, Holcomb JB, Gross KR: The Effect of a Golden Hour Policy on the Morbidity and Mortality of Combat Casualties. JAMA Surg 2016, 151(1):15-24.

24. Davidovic LB, Cinara IS, Ille T, Kostic DM, Dragas MV, Markovic DM: Civil and war peripheral arterial trauma: review of risk factors associated with limb loss. Vascular 2005, 13(3):141-147.

25. Coupland RM: Hand grenade injuries among civilians. JAMA 1993, 270(5):624-626.

26. Muhrbeck M, Holmgren K, Osman Z, von Schreeb J, Wladis A, Andersson P: Trends in Demographics and Surgical Treatment of Weapon-Related Limb Injuries Over Two Decades in a Resource-Scarce Setting. World J Surg 2019, 43(11):2681-2688.

27. Hinsley DE, Rosell PA, Rowlands TK, Clasper JC: Penetrating missile injuries during asymmetric warfare in the 2003 Gulf conflict. Br J Surg 2005, 92(5):637-642.

28. van Gennip L, Haverkamp FJC, Muhrbeck M, Wladis A, Tan E: Using the Red Cross wound classification to predict treatment needs in children with conflict-related limb injuries: a retrospective database study. World J Emerg Surg 2020, 15(1):52.

29. Haac B, Varela C, Geyer A, Cairns B, Charles A: The utility of the Kampala trauma score as a triage tool in a sub-Saharan African trauma cohort. World J Surg 2015, 39(2):356-362.

30. Gardner A, Forson PK, Oduro G, Stewart B, Dike N, Glover P, Maio RF: Diagnostic accuracy of the Kampala Trauma Score using estimated Abbreviated Injury Scale scores and physician opinion. Injury 2017, 48(1):177-183.

31. Institute for Health Metrics and Evaulation (IHME). GBD Compare Data Visualization. Seattle, WA:IHME, University of Washington. Available from http://vizhub.healthdata.org/gbd-compare (Accessed 15 April 2020).

32. Trelles M, Stewart BT, Hemat H, Naseem M, Zaheer S, Zakir M, Adel E, Van Overloop C, Kushner AL: Averted health burden over 4 years at Medecins Sans Frontieres (MSF) Trauma Centre in Kunduz, Afghanistan, prior to its closure in 2015. Surgery 2016, 160(5):1414-1421.

33. Coupland R: Abdominal wounds in war. Br J Surg 1996, 83(11):1505-1511.

34. Aspelund AL, Patel MQ, Kurland L, McCaul M, van Hoving DJ: Evaluating trauma scoring systems for patients presenting with gunshot injuries to a district-level urban public hospital in Cape Town, South Africa. Afr J Emerg Med 2019, 9(4):193-196.

35. Arikan AA, Selcuk E, Bayraktar FA: Predicting Outcomes of Penetrating Cardiovascular Injuries at a Rural Center by Different Scoring Systems. Braz J Cardiovasc Surg 2020, 35(2):198-205.

36. Trunkey DD: Trauma. Accidental and intentional injuries account for more years of life lost in the U.S. than cancer and heart disease. Among the prescribed remedies are improved preventive efforts, speedier surgery and further research. Sci Am 1983, 249(2):28-35.

37. Debarre A: Hard to Reach: Providing Healthcare in Armed Conflict. In.: International Peace Institute; 2018.

38. Wren SM, Wild HB, Gurney J, Amirtharajah M, Brown ZW, Bulger EM, Burkle FM, Jr., Elster EA, Forrester JD, Garber K et al: A Consensus Framework for the Humanitarian Surgical Response to Armed Conflict in 21st Century Warfare. JAMA Surg 2019. 


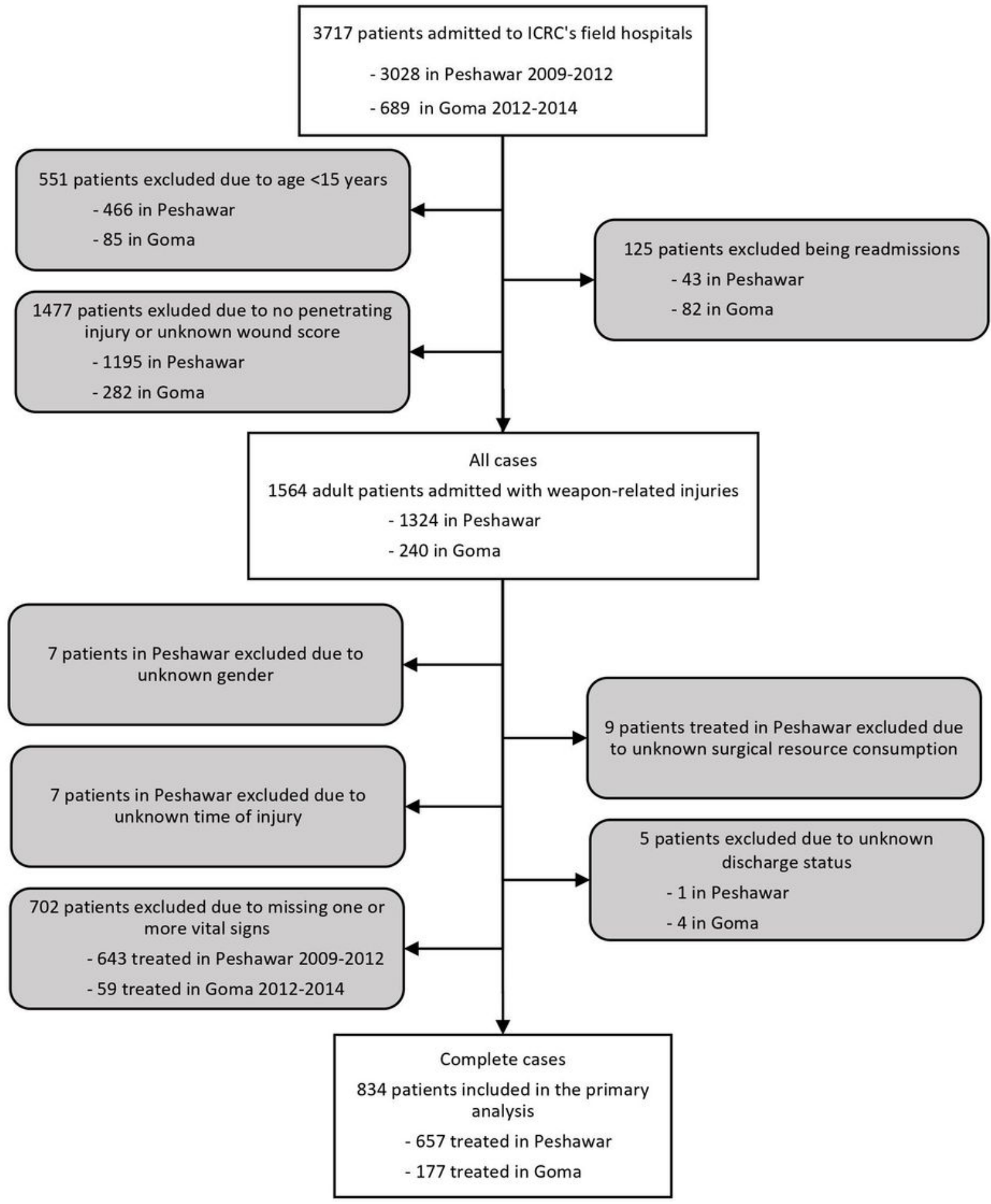

\section{Figure 1}

Flow chart of inclusion. All patients $\geq 15$ years with weapon-related injuries classified with Red Cross Wound Score (RCWS), known surgical resource consumption and discharge status 
a)

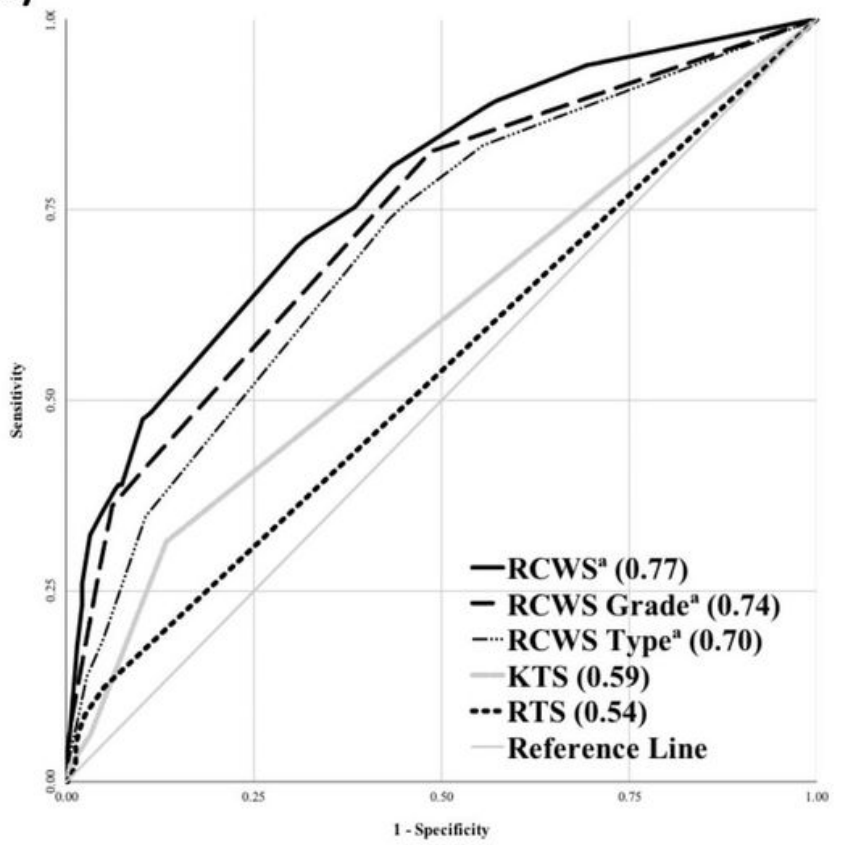

b)

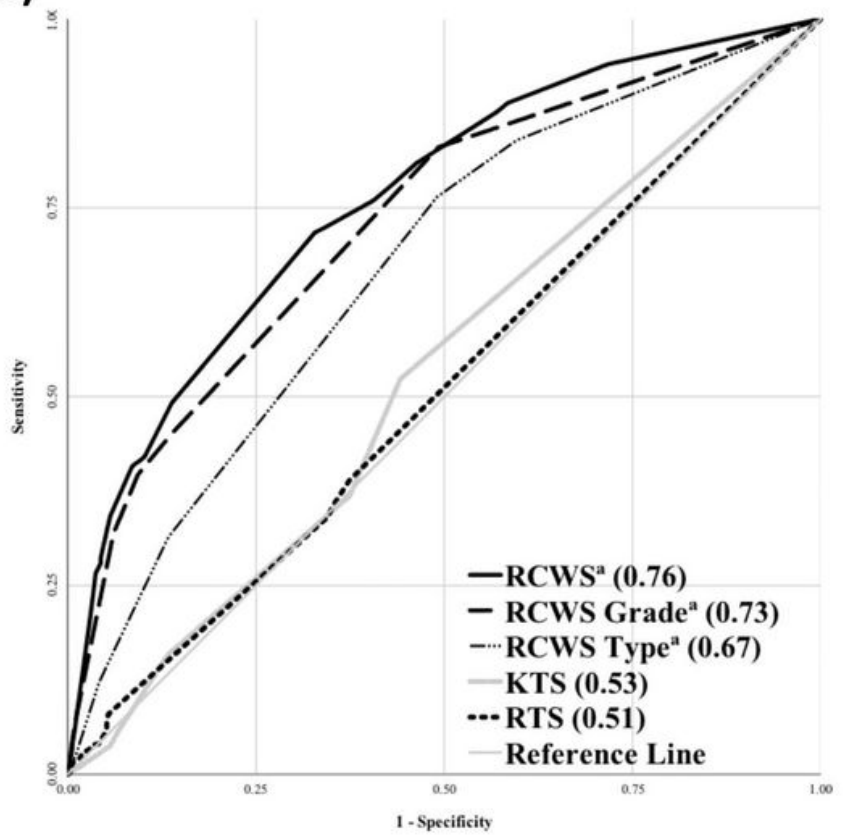

Figure 2

Surgical resource consumption by RCWS, RCWS grade, RCWS type, KTS and RTS (AUC in parenthesis). a) Complete cases $(n=834)$ b) All cases using imputed data for missing variables $(n=1555.9$ patients excluded due to unknown surgical resource consumption) aRCWS, RCWS Grade and RCWS Type was adjusted for existence of several severe injuries (corresponding to Abbreviated Injury Score $\geq 2$ ).

a)

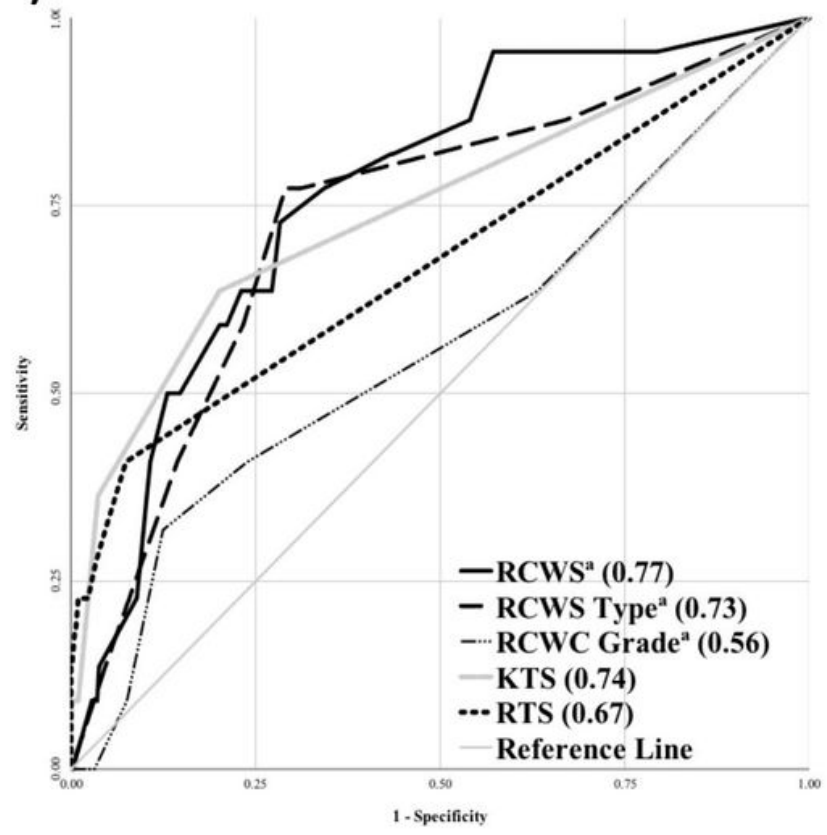

b)

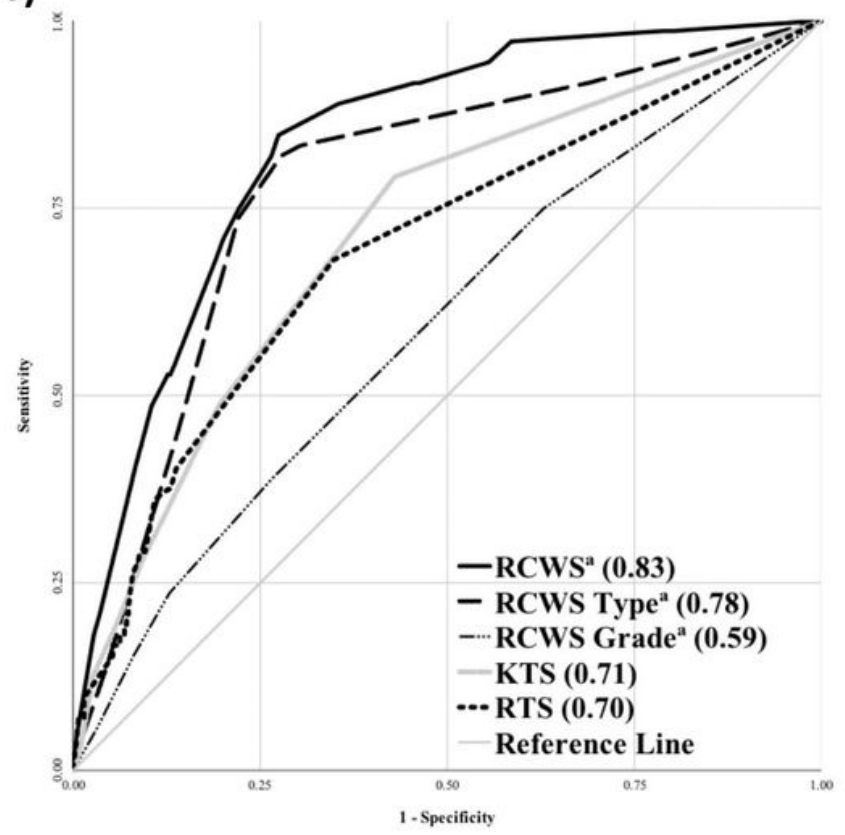

Figure 3 
In-hospital mortality by RCWS, RCWS grade, RCWS type, KTS and RTS (AUC in parenthesis). a) Complete cases $(n=834)$ b) All cases using imputed data for missing variables $(n=1559.5$ patients excluded due to unknown discharge status) aRCWS, RCWS Grade and RCWS Type was adjusted for existence of several severe injuries (corresponding to Abbreviated Injury Score $\geq 2$ ).

\section{Supplementary Files}

This is a list of supplementary files associated with this preprint. Click to download.

- Tables.docx

- RCWCTableSupplEmergMed201212.pdf 